\title{
Errata: A Class of Metric Theories of Gravitation on Minkowski Spacetime
}

\author{
A. Nairz ${ }^{1}$ \\ Received December 2, 1996
}

A mistake which occurred in the derivation of the field equations from the Lagrangian is corrected. Unfortunately many of the expressions given in the article have to be changed, although the results do not change qualitatively, but only quantitatively.

\section{ERRATA}

In a recently published article ${ }^{(1)}$ unfortunately a mistake happened when the linearized field equations for the gravitational potential field were derived from the field Lagrangian. Since these erroneous equations were used as the starting point for all the further investigations, many of the results given in the article have to be corrected, too. The main results and the conclusions that have been drawn, however, remain qualitatively unchanged, although, quantitatively, some expressions depend in a different manner on the parameters of the theory.

The corrected results are given below. They are listed in the same order as they occur in the original article, the equation labels remaining unchanged.

In Sec. 3.1 the linearized field equations, which caused all the trouble, should read

$$
\begin{aligned}
& {\left[\left(1+3 \gamma_{4}\right) \square+\kappa^{2}\right] S_{\mu \nu}+\left(\gamma_{1}-\gamma_{4}-1\right) \partial^{\varrho}\left(S_{\varrho \mu, \nu}+S_{\varrho \nu, \mu}\right)} \\
& \quad+\left(\gamma_{3}-\gamma_{1}\right)\left(\Sigma_{, \mu \nu}+\eta_{\mu \nu} \partial^{\circ} \partial^{\sigma} S_{\varrho \sigma}\right)+\eta_{\mu \nu}\left[\frac{1}{2}\left(\gamma_{1}+\gamma_{2}-2 \gamma_{3}\right) \square+\kappa^{2} \delta\right] \Sigma=0
\end{aligned}
$$

The original factor 2 in front of $\gamma_{4}$ in the first line of (6) is wrong.

\footnotetext{
${ }^{1}$ Institut für Theoretische Physik, Universität Innsbruck, Victor-Franz-Hess-Haus, Technikerstrasse 25/2, A-6020 Innsbruck, Austria.
} 
In the following investigation of the various spin components of pure gravitational fields the correct spin-1 dispersion relation is

$$
k^{0}=\frac{\omega}{c}=\sqrt{\mathbf{k}^{2}+\frac{\kappa^{2}}{\gamma_{1}+2 \gamma_{4}}}
$$

and the range of the spin-1 field is $\kappa / \sqrt{\gamma_{1}+2 \gamma_{4}}$, provided that $\gamma_{1}+2 \gamma_{4}>0$, which is obviously not the case in the version close to general relativity, either. The comments in Sec. 4 of the article on the case of zero spin-1 range, i.e., $\gamma_{1}+2 \gamma_{4}=0$ instead of $\gamma_{1}+\gamma_{4}=0$, remain valid. For the two spin-0 components the explicit expressions for the polynomials $\mathscr{P}, \mathscr{Q}$, which occur in the quadratic equation determining the spin- 0 ranges, are

$$
\begin{aligned}
\mathscr{P} & :=3\left[\gamma_{1} \gamma_{2}-\gamma_{3}\left(\gamma_{3}-1\right)+\gamma_{4}\left(\gamma_{1}+\gamma_{2}+\gamma_{4}-1\right)\right]-\gamma_{1}-\gamma_{2}+\gamma_{3}+\gamma_{4}-1 \\
\mathscr{Q} & :=\gamma_{1}+\gamma_{2}-\gamma_{3}+2 \gamma_{4}-\delta\left(1-3 \gamma_{1}-3 \gamma_{4}\right)
\end{aligned}
$$

The parameters $p$ and $q$ are defined respectively, by

$$
\begin{aligned}
& p:=2 \frac{1+\gamma_{1}-2 \gamma_{3}+\gamma_{4}}{s^{2}+1-\gamma_{1}-\gamma_{3}-\gamma_{4}} \\
& q:=2 \frac{1+\gamma_{1}-2 \gamma_{3}+\gamma_{4}}{\sigma^{2}+1-\gamma_{1}-\gamma_{3}-\gamma_{4}}
\end{aligned}
$$

the denominators of which should not become zero, i.e., $s^{2} \neq \gamma_{1}+\gamma_{3}+$ $\gamma_{4}-1$ and $\sigma^{2} \neq \gamma_{1}+\gamma_{3}+\gamma_{4}-1$, respectively.

In Sec. 3.2 the first field equation for static, spherically and timeinversal symmetric fields remains unaltered, and the second and third should read

$$
\begin{aligned}
\left(\gamma_{1}+\gamma_{2}-2 \gamma_{3}\right) \tilde{U}^{\prime \prime}+\left(\gamma_{2}-\gamma_{3}\right) \frac{2}{r} \tilde{U}^{\prime}-2 \kappa^{2} \delta \tilde{U} \\
\quad+\left(\gamma_{2}-\gamma_{1}\right) \tilde{V}^{\prime \prime}-\left(2+2 \gamma_{1}-\gamma_{2}-3 \gamma_{3}+2 \gamma_{4}\right) \frac{2}{r} \tilde{V}^{\prime} \\
\quad-\left(\frac{4\left(1-\gamma_{1}-\gamma_{3}-\gamma_{4}\right)}{r^{2}}+2 \kappa^{2} \delta\right)(\tilde{V}-\tilde{W}) \\
\quad+2\left(1+\gamma_{1}+\gamma_{2}-2 \gamma_{3}+3 \gamma_{4}\right)\left(\tilde{W}^{\prime \prime}+\frac{2}{r} \tilde{W}^{\prime}\right)-2(1+3 \delta) \kappa^{2} \tilde{W}=0
\end{aligned}
$$




$$
\begin{aligned}
& \left(\gamma_{1}-\gamma_{3}\right)\left(\tilde{U}^{\prime \prime}-\frac{1}{r} \tilde{U}^{\prime}\right)+\left(1-\gamma_{1}-\gamma_{3}-\gamma_{4}\right) \tilde{V}^{\prime \prime} \\
& -\left(3 \gamma_{1}-\gamma_{3}+4 \gamma_{4}\right) \frac{\tilde{V}^{\prime}}{r}+\left(\frac{2\left(4 \gamma_{1}+5 \gamma_{4}-1\right)}{r^{2}}+\kappa^{2}\right)(\tilde{V}-\tilde{W}) \\
& +\left(1+2 \gamma_{1}-2 \gamma_{3}+3 \gamma_{4}\right) \tilde{W}^{\prime \prime}+\left(\gamma_{1}+\gamma_{3}+\gamma_{4}-1\right) \frac{2}{r} \tilde{W}^{\prime}=0
\end{aligned}
$$

Fortunately the contents of the following Sects. 3.3-3.5 is still correct if one uses the corrected values for $p, q$, etc.

The most important changes have to be made in Sec. 3.6, where the possible sets of parameters compatible with the so-called "solar-system tests" were determined. Similar to the original version, $\gamma_{0}, \gamma_{3}$, and $\gamma_{4}$ depend on $\gamma_{1}, \gamma_{2}$, and $\delta$, but in a modified manner:

$$
\gamma_{0}=\frac{1}{2\left(1-3 \gamma_{1}-3 \gamma_{4}\right)} \quad\left(\gamma_{1}+\gamma_{4} \neq \frac{1}{3}\right)
$$

from which follows $\gamma_{1}=-2 \gamma_{4}$ or $\gamma_{4}=-\frac{1}{2} \gamma_{1}$, and one of the two possibilities

$$
\gamma_{3}=\frac{2+\gamma_{1}}{4} \quad \text { or } \quad \gamma_{3}=1-\frac{1}{2} \gamma_{1}
$$

The condition $\gamma_{4}>-\frac{1}{3}$ of Sec. 3.1 then has as the consequence that $\gamma_{1}<\frac{2}{3}$. Further investigation reveals the fact that the choice $\gamma_{3}=\frac{1}{4}\left(2+\gamma_{1}\right)$ fails to allow $\operatorname{sign}(\mathscr{P})=\operatorname{sign}(\mathscr{2})=\operatorname{sign}(1+4 \delta)$, and thus $\gamma_{3}=1-\frac{1}{2} \gamma_{1}$ is the only possibility; the condition that $s^{2} \neq \gamma_{1}+\gamma_{3}+\gamma_{4}-1 \neq \sigma^{2}$ then becomes redundant, since $s^{2} \neq 0 \neq \sigma^{2}$ has always been presupposed.

The equivalent of Fig. 1, i.e., the maximum allowed subset of the parameters $\gamma_{1}$ and $\gamma_{2}$, provided that $-1<\delta<-\frac{1}{4}$ applies, looks qualitatively the same, but is bounded by the straight lines $\gamma_{2}-\gamma_{1}=0(\Leftrightarrow \mathscr{P}=0$ and $\mathscr{Q}(\delta=-1)=0$, respectively) and $\gamma_{2}+\frac{1}{8} \gamma_{1}=\frac{3}{4}\left(\Leftrightarrow \mathscr{2}\left(\delta=-\frac{1}{4}\right)=0\right)$. The region $\mathscr{2}^{2}-(1+4 \delta) \mathscr{P}>0$ is bounded by

$\gamma_{2}-\frac{1}{4}(1+6 \delta \pm 3 \sqrt{-3(1+4 \delta)}) \cdot \gamma_{1}+\frac{1}{2}(2 \delta-1 \pm \sqrt{-3(1+4 \delta)})=0$

The correct summary at the end of Sec. 3.6 concerning the parameters therefore is 
1. Dependent on the choice of $\delta \in\left(-1,-\frac{1}{4}\right), \gamma_{1}$ and $\gamma_{2}$ can take on any value in the wedge-shaped intersection of the open half-planes

$\gamma_{2}>\gamma_{1}$ and $\gamma_{2}<\frac{1}{4}(1+6 \delta+3 \sqrt{-3(1+4 \delta)}) \cdot \gamma_{1}-\frac{1}{2}(2 \delta-1+\sqrt{-3(1+4 \delta)})$

2. $\gamma_{0}=1 /\left(2-3 \gamma_{1}\right)$

3. $\gamma_{3}=1-\frac{1}{2} \gamma_{1}$

4. $\gamma_{4}=-\frac{1}{2} \gamma_{1}$

As an appropriate example, then, take $\delta=-\frac{1}{3}$ and $\gamma_{1}=-2, \gamma_{2}=-1$. We get $\gamma_{0}=1 / 8, \gamma_{3}=2, \gamma_{4}=1$; the ranges are $\mu_{1}^{2}=\kappa^{2} / 4, \mu_{2}^{2}=\kappa^{2} / s^{2}$ with $s^{2}=5-\sqrt{13}$ and $\mu_{3}^{2}=\kappa^{2} / \sigma^{2}$ with $\sigma^{2}=5+\sqrt{13}$.

In Sec. 4, taking into account the results obtained above, the linearized field equations for the gravitational potential field coupled to a conserved nongravitational energy-momentum tensor $T_{\mu \nu}$ should read

$$
\begin{gathered}
\frac{1}{2}\left(2-3 \gamma_{1}\right)\left\{\square S_{\mu v}-\partial^{\varrho}\left(S_{\varrho \mu, v}+S_{\varrho v, \mu}\right)+\Sigma_{, \mu \nu}+\eta_{\mu \nu}\left(\partial^{\varrho} \partial^{\sigma} S_{\varrho \sigma}-\square \Sigma\right)\right\} \\
+\kappa^{2} S_{\mu v}+\eta_{\mu v}\left(\frac{1}{2}\left(\gamma_{2}-\gamma_{1}\right) \square+\kappa^{2} \delta\right) \Sigma=\varepsilon T_{\mu v}
\end{gathered}
$$

where comparison with statics yields $\varepsilon=-4 \pi G_{N}\left(2-3 \gamma_{1}\right) / c^{4}$.

The last change concerns the explicit expression for the gravitational Lagrangian, $\mathscr{L}_{s}$, in the Summary and Conclusion (Sec. 5). The possible class of Lagrangians that is able to stand the "solar-system tests" is

$$
\mathscr{L}_{s}=\frac{c^{3}}{8 \pi G_{N}} \frac{P^{n}}{2-3 \gamma_{1}}\left\{T_{0}+T_{3}+\gamma_{1}\left(T_{1}-\frac{1}{2} T_{3}-\frac{1}{2} T_{4}\right)+\gamma_{2} T_{2}-\kappa^{2} V_{0}-\kappa^{2} \delta V_{1}\right\}
$$

where $\gamma_{1}$ and $\gamma_{2}$ can be arbitrarily chosen in the $\delta$-dependent domain given above.

\section{REFERENCE}

1. A. Nairz, Found. Phys. 26(3), 369-389 (1996). 